\title{
Subtypes of Mania Determined by Grade of Membership Analysis
}

\author{
Frederick Cassidy, M.D., Carl F. Pieper, M.P.H., D.P.H., and Bernard J. Carroll, M.B., Ph.D.
}

Classical descriptions of mania subtypes extend back to Kraepelin; however, in marked contrast to the study of depression subtypes, validation of mania subtypes by multivariate statistical methods has seldom been attempted. We applied Grade of Membership (GOM) analysis to the rated clinical features of 327 inpatients with DSM-III-R mania diagnoses. GOM is a type of latent structure multivariate analysis, which differs from others of this type in making no a priori distributional assumptions about groupings. We obtained 5 GOM Pure Types with good face validity. The major Kraepelinian forms of "hypomania," "acute mania," "delusional mania," and "depressive or anxious mania" were validated. The major new finding is of two mixed mania presentations, each with marked lability of mood. The first of these displayed a dominant mood of severe depression with labile periods of pressured, irritable hostility and paranoia, and the complete absence of euphoria or humor. The second mixed mania Pure Type displayed a true, incongruous mixture of affects: periods of classical manic symptoms with euphoria, elation, humor, grandiosity, psychosis, and psychomotor activation, switching frequently to moderately depressed mood with pressured anxiety and irritability. This multivariate analysis validated classical clinical descriptions of the major subtypes of mania. Two distinct forms of mixed manic episodes were identified. DSM-III-R criteria did not reliably identify either of these two natural groups of mixed bipolar patients. As occurs in depression, this clinical heterogeneity of mania may influence response to drug treatments.

[Neuropsychopharmacology 25:373-383, 2001] (C) 2001 American College of Neuropsychopharmacology. Published by Elsevier Science Inc.
KEY WORDS: Mania; Subtypes; Mixed states; Kraepelin; Diagnosis; Grade of membership classification

A century ago, following the acceptance of manic-depressive illness as a nosologic construct, the subtyping of manic episodes was first addressed. Robertson proposed two varieties of acute mania: furious or raging mania and

From the Duke-Umstead Bipolar Disorders Program (FC) Department of Psychiatry and Behavioral Sciences, Duke University, Durham, North Carolina; Center for the Study of Aging and Human Development (CFP), Department of Community and Family Medicine, Duke University Medical Center, Durham, North Carolina; Pacific Behavioral Research Foundation (BJC), Carmel, California, USA

Address correspondence to: Dr. F. Cassidy, Duke-Umstead Bipolar Disorders Program, Box 3414 DUMC, Durham, NC 27710. Tel.: 919-575-7801; Fax: 919-575-4069; E-mail: cassi002@mc.duke.edu

Received August 8, 2000; revised November 17, 2000; accepted January 8, 2001. hilarious mania (Robertson 1890). Weygandt suggested dividing manic-depressive episodes into manic, depressed, and mixed states (Weygandt 1901). Kraepelin also divided manic-depressive disorder into manic, depressed, and mixed states (Kraepelin 1921). He went on to develop an elaborate nosology of three manic subtypes (hypomania, acute mania, and delirious mania) and no fewer than six mixed subtypes (depressive or anxious mania, excited depression, mania with poverty of thought, manic stupor, depression with flight of ideas, and inhibited mania). Most of these mixed or transitional manic-depressive states were predicated on varying admixtures of three basic elements: mood, psychic activity, and motor activity (Sims 1988; Goodwin and Jamison 1990).

The study of manic subtypes then remained essentially dormant until 1969, when Winokur, Clayton, and Reich (Winokur et al. 1969) reported on the high fre- 
quency of depressive symptoms in mania. They set an arbitrary standard of diagnostic criteria for mixed bipolar episodes (corresponding to Kraepelin's anxious or depressive mania) by requiring the full depressive as well as the full manic syndrome. Without clear validation, that standard was adopted by the American Psychiatric Association in DSM-III, DSM-III-R, and DSM-IV. We and others have called attention to problems with the DSM criteria for mixed bipolar episode in recent publications (McElroy et al. 1992; Cassidy et al. 1997). The other manic and mixed bipolar subtypes proposed by Kraepelin were never addressed in the DSM classifications.

In marked contrast to the intensive study and classification of depressive episodes (Kendell 1976), the subtyping of manic episodes by multivariate statistical methods has been generally neglected. A few years after publication of the now classic volume of Winokur, Clayton, and Reich (1969), some investigators proposed a statistical typology of euphoric-grandiose (EG) and paranoid-destructive (PD) mania (Beigel and Murphy 1971; Murphy and Beigel 1974). By factor analysis, depressive symptoms were associated with the PD symptoms. This view ignored the work of Winokur, Clayton, and Reich (1969),, but it became quite influential and found its way into many textbooks. As we have previously discussed (Cassidy et al. 1998a), the clinical samples used in those studies were inadequate.

Our own factor analysis of symptoms rated in 237 manic patients by the Scale for Manic States (SMS, Cassidy et al. 1998a, 1998b), did not confirm the EG and PD factors of Beigel, Murphy, and Double (Beigel and Murphy 1971; Murphy and Beigel 1974; Double 1990). Rather, we found five distinct, orthogonal factors that represent key clinical domains of mania; namely, (1) depression and dysphoric mood; (2) psychomotor acceleration; (3) psychosis; (4) hedonic activation; and (5) irritable aggression. The first factor comprised the symptoms depressed mood, guilt feelings, anxiety, lability, and suicidal tendency. This dysphoria factor was quite distinct from the factor comprising irritable aggression. The distribution of scores on the dysphoria factor was bimodal, which suggested that a distinct group of mixed manic episodes could be identified. Dilsaver et al. (1999) confirmed our key findings that paranoia and aggression comprise a factor distinct from depressed mood in manic patients and that the distribution of scores on the depression factor is bimodal.

As we earlier proposed (Cassidy et al. 1998b) we now approach the issue of mania subtypes with a powerful multivariate statistical technique, Grade of Membership (GOM) analysis. A general description of GOM and its associated principles is available (Swartz et al. 1986). Briefly, GOM is a type of latent structure analysis, but different from others of this type in making no a priori distributional assumptions about groupings. Rather than assume discrete latent classes, GOM assumes "fuzzy sets" for which the degrees of membership can be specified both for internal variables (signs and symptoms) and for external variables thought to be related, but not causative of the internal structures (e.g., demographic characteristics). Thus, individual variables may be more or less related to a pure type, and individual patients may demonstrate partial membership in more than one group. The statistically defined symptom profiles are termed Pure Types. As recently discussed by Nurnberg et al. (1999), two Pure Types may represent different disease processes or the same disease process that is expressed differently because of different patient attributes or different stages in the progression of a disease. By maximum likelihood estimates, complementary coefficients of loading by subjects on the Pure Types are derived. The subject's degree or grade of membership in a Pure Type is denoted by a coefficient of association, $g_{i k}$, which defines the degree to which the $\mathrm{i}^{\text {th }}$ person belongs to the $\mathrm{k}^{\text {th }}$ Pure Type. This coefficient may range from 0 to 1.0. A subject may be a member of one Pure Type exclusively but more commonly will have partial membership in more than one Pure Type. Each internal variable used to determine the GOM Pure Types has a calculated coefficient, $k_{\mathrm{j} 1}$ that describes each of the $k$ Pure Types in terms of the probability that it will have the $1^{\text {th }}$ response to the $\mathrm{j}^{\text {th }}$ variable. These coefficients also lie between 0 and 1.0. After the symptom profiles representing the GOM Pure Types are defined, subjects are then allocated to GOM groups according to their predominant $g_{i k}$ loadings, as described in Methods. To recapitulate, a GOM Pure Type is a symptom profile; whereas, a GOM group is a subset of the cohort of subjects.

\section{METHODS}

We studied 327 adult inpatients at John Umstead Hospital (JUH), a state psychiatric hospital serving 16 counties in north-central North Carolina. All met DSM-III-R criteria and carried clinical diagnoses of bipolar disorder, manic or mixed. In most cases, the manic episode led to involuntary commitment to inpatient status. Clinical features were evaluated with the Scale for Manic States (SMS, Cassidy et al. 1998b). This scale comprises 15 classic features of mania and five features relevant to mixed bipolar states. Each sign or symptom is rated from 0 to 5 in severity, with descriptive anchor statements provided for scores of 1,3, and 5. Ratings were completed based on patient interview, chart review, and observation on the ward. Further details of the rating procedure and on the validity and reliability of the SMS are presented elsewhere (Cassidy et al. 1998a, 1998b).

\section{Study Cohort}

The 327 patients included 156 males and 171 females. There were $284(86.9 \%)$ meeting DSM-III-R criteria for bipolar disorder, manic episode, and $43(13.1 \%)$ diagnosed bipolar disorder, mixed episode. The mean age was 42.2 , 
SD 14.0 years, with a range from 18 to 82 . The ethnic composition of the sample was 188 whites, 137 African Americans, 1 Asian American, and 1 Native American. The mean total symptom score on the SMS was 34.7, SD 9.8 , with a range of 11 to 59 . When patients were evaluated in more than one episode, only ratings from the first of these episodes are included in this report.

\section{Statistical Analysis}

To obtain the maximum resolution, we structured the GOM analysis to derive six values for each internal variable, one for each level of severity (0 to 5) of the features rated on the SMS. GOM analyses were performed assuming from two to seven Pure Types. The log likelihood ratio describes the goodness of fit for each typologic solution. A chi-squared analysis was performed to test the hypothesis that additional Pure Types did not significantly improve the fit of the model to the data and was used to determine the appropriate number of Pure Types. In determining GOM groups, subjects were assigned according to the Pure Type for which the quotient of the subject's $g_{i k}$ divided by the mean $g_{i k}$ of all subjects for that Pure Type was highest as described elsewhere. The average $g_{\text {ik }}$ for each of the five Pure Types was computed by forced group assignment, and the frequency the Pure Type characteristic within each forced group was calculated as the product of the number of subjects assigned to the group and the mean $\mathrm{g}_{\mathrm{ik}}$.

Subscale severity scores for each of the five factors of the SMS were computed for each subject as the sums of the ratings of each feature that loaded significantly on each factor (Cassidy et al. 1998c). These five factor subscales are: (1) dysphoria rated as depressed mood, anxiety, lability of mood, guilt feelings, and suicidal tendency; (2) psychomotor acceleration rated as increased motor activity, pressured speech, racing thoughts, and intrusiveness; (3) psychosis rated as any delusions or hallucinations, paranoid thoughts, delusional grandiosity, and delusional denial of illness; (4) hedonic activation rated as euphoric and expansive mood, grandiosity, humor, and increased sexuality; and (5) irritable aggression rated as irritability, aggression, and paranoid hostility. An analysis of variance with post-hoc Tukey tests was conducted comparing mean scores on each of the 5 SMS subscales across the final number of Pure Types. The principal external variable was the classification of subjects as manic episode or mixed episode by DSM-III-R criteria. Each final GOM group was examined for association with this clinical classification using $\chi^{2}$ analysis.

\section{RESULTS}

In the GOM analyses, goodness of fit improved significantly up to the transition from four to five Pure Types $\left(\chi^{2}=556.097, \mathrm{df}=427, p<.001\right)$, but not in the change from five to six Pure Types $\left(\chi^{2}=449.842, \mathrm{df}=427\right.$, ns). Five Pure Types, therefore, were determined. Lambda coefficients for each of the 20 signs and symptoms scored 0 to 5 are given in Table 1. The GOM group assignments indicated that each Pure Type was strongly represented (Table 2). The most common was Group 2 at $24.5 \%$ of the sample, and the least common was Group 5 at $15.6 \%$ of the sample. Average scores on the SMS-rated Factor 1 (dysphoria), and on the nondysphoric severity scale that includes all the signs and symptoms from factors 2-5 (Cassidy et al. 1998c) are shown in Table 2. Scores on all SMS factors as well as analysis of variance (ANOVA) by GOM groups are shown in Table 3. From the profile of symptom loadings and factor scores in Tables 1, 2, and 3, the five Pure Types can be described.

Pure Type 1 appears as the mildest in severity on all measures. Pure Type 1 represents relatively mild, classic manic episodes that completely lack dysphoric or paranoid or aggressive features. They have low psychosis ratings and minimal lability of mood. They have moderate psychomotor acceleration and notable hedonic activation.

Pure Type 2 appears as the most severe on the classical manic factors 2-5 but is essentially devoid of dysphoric features. Pure Type 2 episodes have the highest scores for 13 of the 15 classical manic symptoms (irritability and aggression are the only exceptions). They are markedly disturbed in their sleep, very pressured, very psychotic, paranoid, grandiose, funny, hypersexual, intrusive and lacking in insight.

Pure Type 3 episodes are characterized by high ratings of grandiosity and psychosis but without the marked psychomotor pressure, sleep disturbance, hypersexuality, humor, and irritable paranoia of Pure Type 2 episodes. Pure Type 3 episodes also are essentially devoid of dysphoric features, but there is a slight increase of mood lability relative to Pure Types 1 and 2.

Pure Type 4 episodes are quite different from Pure Types 1-3 in having very high ratings on all symptoms of the dysphoria factor and by far the highest total score on SMS Factor 1. Pure Type 4 episodes are marked by a complete absence of euphoric mood or humor and minimal ratings of grandiosity or hypersexuality. They have by far the lowest scores on SMS Factor 4, hedonic activation. They have high levels of aggression, irritability, and psychosis, including paranoia. Pure Type 4 episodes have overall low scores on the nonmixed SMS symptoms.

Pure Type 5 episodes also differ from Pure Types 1-3 by their moderately high ratings on the dysphoria factor. At the same time, they are significantly less dysphoric than Pure Type 4 episodes. They have elevated scores on depressed mood, anxiety, and lability, and slight suicidal ideation. Unlike Pure Type 4 episodes, however, they have higher levels of grandiosity, humor, sexuality, and psychomotor activation. They are 
Table 1. $\lambda$ Values for Scale for Manic States Item Scores (Scale 0-5)

\begin{tabular}{|c|c|c|c|c|c|c|}
\hline \multirow[b]{2}{*}{ Sign/symptom } & \multirow{2}{*}{$\begin{array}{l}\text { SMS } \\
\text { score }\end{array}$} & \multicolumn{5}{|c|}{ Pure types } \\
\hline & & 1 & 2 & 3 & 4 & 5 \\
\hline \multirow[t]{6}{*}{ Increased motor activity } & 0 & 0.0000 & 0.0000 & 0.0000 & 0.2408 & 0.0000 \\
\hline & 1 & 0.0000 & 0.0000 & 0.2775 & 0.0504 & 0.1942 \\
\hline & 2 & 0.3112 & 0.0000 & 0.7225 & 0.2631 & 0.0000 \\
\hline & 3 & 0.6888 & 0.2776 & 0.0000 & 0.4241 & 0.4973 \\
\hline & 4 & 0.0000 & 0.6398 & 0.0000 & 0.0000 & 0.2592 \\
\hline & 5 & 0.0000 & 0.0827 & 0.0000 & 0.0215 & 0.0493 \\
\hline \multirow[t]{6}{*}{ Decreased sleep } & 0 & 0.1684 & 0.0000 & 0.0997 & 0.1110 & 0.0000 \\
\hline & 1 & 0.1382 & 0.0000 & 0.3939 & 0.0000 & 0.0000 \\
\hline & 2 & 0.0000 & 0.0000 & 0.5063 & 0.0000 & 0.0000 \\
\hline & 3 & 0.2280 & 0.0000 & 0.0000 & 0.3649 & 0.7172 \\
\hline & 4 & 0.3810 & 0.7442 & 0.0000 & 0.5240 & 0.2828 \\
\hline & 5 & 0.0843 & 0.2558 & 0.0000 & 0.0000 & 0.0000 \\
\hline \multirow[t]{6}{*}{ Pressured speech } & 0 & 0.0421 & 0.0000 & 0.0000 & 0.3335 & 0.0000 \\
\hline & 1 & 0.1329 & 0.0000 & 0.4167 & 0.1079 & 0.0000 \\
\hline & 2 & 0.1519 & 0.0000 & 0.5833 & 0.3000 & 0.0000 \\
\hline & 3 & 0.6731 & 0.0971 & 0.0000 & 0.2586 & 0.3396 \\
\hline & 4 & 0.0000 & 0.6901 & 0.0000 & 0.0000 & 0.6604 \\
\hline & 5 & 0.0000 & 0.2128 & 0.0000 & 0.0000 & 0.0000 \\
\hline \multirow{6}{*}{ Racing thoughts/disturbed concentration } & 0 & 0.1364 & 0.0637 & 0.0000 & 0.0515 & 0.0000 \\
\hline & 1 & 0.1034 & 0.0375 & 0.3813 & 0.1075 & 0.1420 \\
\hline & 2 & 0.3344 & 0.0000 & 0.4092 & 0.2539 & 0.1937 \\
\hline & 3 & 0.4258 & 0.2502 & 0.2095 & 0.4877 & 0.1001 \\
\hline & 4 & 0.0000 & 0.5399 & 0.0000 & 0.0995 & 0.4645 \\
\hline & 5 & 0.0000 & 0.1088 & 0.0000 & 0.0000 & 0.0997 \\
\hline \multirow[t]{6}{*}{ Mood lability } & 0 & 0.8433 & 0.5536 & 0.0000 & 0.0000 & 0.0000 \\
\hline & 1 & 0.1567 & 0.3052 & 0.6288 & 0.1818 & 0.0000 \\
\hline & 2 & 0.0000 & 0.0537 & 0.3712 & 0.0000 & 0.2692 \\
\hline & 3 & 0.0000 & 0.0432 & 0.0000 & 0.3399 & 0.4642 \\
\hline & 4 & 0.0000 & 0.0000 & 0.0000 & 0.4086 & 0.2666 \\
\hline & 5 & 0.0000 & 0.0444 & 0.0000 & 0.0697 & 0.0000 \\
\hline \multirow[t]{6}{*}{ Euphoric mood } & 0 & 0.0000 & 0.0000 & 0.0000 & 1.0000 & 0.0000 \\
\hline & 1 & 0.1694 & 0.0000 & 0.0000 & 0.0000 & 0.7472 \\
\hline & 2 & 0.0000 & 0.0000 & 1.0000 & 0.0000 & 0.1904 \\
\hline & 3 & 0.6306 & 0.4430 & 0.0000 & 0.0000 & 0.0000 \\
\hline & 4 & 0.2000 & 0.3942 & 0.0000 & 0.0000 & 0.0624 \\
\hline & 5 & 0.0000 & 0.1628 & 0.0000 & 0.0000 & 0.0000 \\
\hline \multirow[t]{6}{*}{ Dysphoric mood } & 0 & 1.0000 & 1.0000 & 0.6645 & 0.0000 & 0.0000 \\
\hline & 1 & 0.0000 & 0.0000 & 0.3355 & 0.0877 & 0.2570 \\
\hline & 2 & 0.0000 & 0.0000 & 0.0000 & 0.0899 & 0.3893 \\
\hline & 3 & 0.0000 & 0.0000 & 0.0000 & 0.6107 & 0.3536 \\
\hline & 4 & 0.0000 & 0.0000 & 0.0000 & 0.2118 & 0.0000 \\
\hline & 5 & 0.0000 & 0.0000 & 0.0000 & 0.0000 & 0.0000 \\
\hline \multirow[t]{6}{*}{ Guilt } & 0 & 1.0000 & 1.0000 & 1.0000 & 0.3946 & 0.9463 \\
\hline & 1 & 0.0000 & 0.0000 & 0.0000 & 0.3107 & 0.0000 \\
\hline & 2 & 0.0000 & 0.0000 & 0.0000 & 0.0754 & 0.0537 \\
\hline & 3 & 0.0000 & 0.0000 & 0.0000 & 0.2011 & 0.0000 \\
\hline & 4 & 00.000 & 0.0000 & 0.0000 & 0.0183 & 0.0000 \\
\hline & 5 & 0.0000 & 0.0000 & 0.0000 & 0.0000 & 0.0000 \\
\hline \multirow[t]{6}{*}{ Suicide } & 0 & 1.0000 & 1.0000 & 1.0000 & 0.4227 & 0.6189 \\
\hline & 1 & 0.0000 & 0.0000 & 0.0000 & 0.2162 & 0.2320 \\
\hline & 2 & 0.0000 & 0.0000 & 0.0000 & 0.1825 & 0.0860 \\
\hline & 3 & 0.0000 & 0.0000 & 0.0000 & 0.1072 & 0.0000 \\
\hline & 4 & 0.0000 & 0.0000 & 0.0000 & 0.0536 & 0.0000 \\
\hline & 5 & 0.0000 & 0.0000 & 0.0000 & 0.0179 & 0.0000 \\
\hline \multirow[t]{6}{*}{ Psychosis } & 0 & 0.2750 & 0.0000 & 0.0000 & 0.1155 & 0.0000 \\
\hline & 1 & 0.5294 & 0.0000 & 0.0000 & 0.0000 & 0.0000 \\
\hline & 2 & 0.0000 & 0.0000 & 0.1654 & 0.0000 & 0.1862 \\
\hline & 3 & 0.1022 & 0.0000 & 0.0000 & 0.1360 & 0.5258 \\
\hline & 4 & 0.0934 & 0.5266 & 0.6059 & 0.7485 & 0.2880 \\
\hline & 5 & 0.0000 & 0.4734 & 0.2287 & 0.0000 & 0.0000 \\
\hline
\end{tabular}


Table 1. (continued)

\begin{tabular}{|c|c|c|c|c|c|c|}
\hline \multirow[b]{2}{*}{ Sign/symptom } & \multirow{2}{*}{$\begin{array}{l}\text { SMS } \\
\text { score }\end{array}$} & \multicolumn{5}{|c|}{ Pure types } \\
\hline & & 1 & 2 & 3 & 4 & 5 \\
\hline \multirow[t]{6}{*}{ Paranoia } & 0 & 1.0000 & 0.0000 & 0.0000 & 0.0000 & 0.0000 \\
\hline & 1 & 0.0000 & 0.0823 & 0.3289 & 0.2272 & 0.0000 \\
\hline & 2 & 0.0000 & 0.0000 & 0.4483 & 0.0000 & 0.0000 \\
\hline & 3 & 0.0000 & 0.0000 & 0.0000 & 0.0000 & 1.0000 \\
\hline & 4 & 0.0000 & 0.6105 & 0.0000 & 0.6911 & 0.0000 \\
\hline & 5 & 0.0000 & 0.3072 & 0.2228 & 0.0817 & 0.0000 \\
\hline \multirow[t]{6}{*}{ Grandiosity } & 0 & 0.1528 & 0.0000 & 0.0000 & 0.6779 & 0.0000 \\
\hline & 1 & 0.2992 & 0.0000 & 0.0000 & 0.2041 & 0.0887 \\
\hline & 2 & 0.2598 & 0.0000 & 0.3299 & 0.1180 & 0.0000 \\
\hline & 3 & 0.2881 & 0.0000 & 0.0000 & 0.0000 & 0.9113 \\
\hline & 4 & 0.0000 & 0.7036 & 0.5398 & 0.0000 & 0.0000 \\
\hline & 5 & 0.0000 & 0.2964 & 0.1303 & 0.0000 & 0.0000 \\
\hline \multirow[t]{6}{*}{ Lack of insight } & 0 & 0.3264 & 0.0000 & 0.0000 & 0.1948 & 0.0000 \\
\hline & 1 & 0.4333 & 0.0000 & 0.0000 & 0.3396 & 0.1835 \\
\hline & 2 & 0.1425 & 0.0992 & 0.2729 & 0.1585 & 0.0000 \\
\hline & 3 & 0.0979 & 0.2592 & 0.1095 & 0.1034 & 0.3727 \\
\hline & 4 & 0.0000 & 0.3069 & 0.6176 & 0.0877 & 0.4438 \\
\hline & 5 & 0.0000 & 0.3347 & 0.0000 & 0.1161 & 0.0000 \\
\hline \multirow{6}{*}{ Increased contact } & 0 & 0.0000 & 0.0000 & 0.3448 & 0.4215 & 0.0000 \\
\hline & 1 & 0.5222 & 0.0000 & 0.3002 & 0.2688 & 0.0000 \\
\hline & 2 & 0.3523 & 0.0000 & 0.3550 & 0.3097 & 0.4247 \\
\hline & 3 & 0.1256 & 0.5400 & 0.0000 & 0.0000 & 0.3944 \\
\hline & 4 & 0.0000 & 0.3545 & 0.0000 & 0.0000 & 0.1809 \\
\hline & 5 & 0.0000 & 0.1055 & 0.0000 & 0.0000 & 0.0000 \\
\hline \multirow[t]{6}{*}{ Increased sexuality } & 0 & 0.0870 & 0.2446 & 0.6563 & 0.9318 & 0.2083 \\
\hline & 1 & 0.1898 & 0.1303 & 0.3009 & 0.0000 & 0.4431 \\
\hline & 2 & 0.3613 & 0.0000 & 0.0000 & 0.0000 & 0.2766 \\
\hline & 3 & 0.2614 & 0.3196 & 0.0000 & 0.0000 & 0.0000 \\
\hline & 4 & 0.1004 & 0.3055 & 0.0000 & 0.0682 & 0.0000 \\
\hline & 5 & 0.0000 & 0.0000 & 0.0428 & 0.0000 & 0.0000 \\
\hline \multirow[t]{6}{*}{ Humor } & 0 & 0.0272 & 0.4391 & 0.6287 & 1.0000 & 0.3323 \\
\hline & 1 & 0.5396 & 0.1552 & 0.2668 & 0.0000 & 0.5328 \\
\hline & 2 & 0.3323 & 0.2726 & 0.0000 & 0.0000 & 0.0000 \\
\hline & 3 & 0.0000 & 0.0759 & 0.1045 & 0.0000 & 0.1349 \\
\hline & 4 & 0.1008 & 0.0426 & 0.0000 & 0.0000 & 0.0000 \\
\hline & 5 & 0.0000 & 0.0146 & 0.0000 & 0.0000 & 0.0000 \\
\hline \multirow[t]{6}{*}{ Anxiety } & 0 & 1.0000 & 1.0000 & 0.8759 & 0.0000 & 0.0000 \\
\hline & 1 & 0.0000 & 0.0000 & 0.1241 & 0.1066 & 0.6398 \\
\hline & 2 & 0.0000 & 0.0000 & 0.0000 & 0.2185 & 0.3602 \\
\hline & 3 & 0.0000 & 0.0000 & 0.0000 & 0.4602 & 0.0000 \\
\hline & 4 & 0.0000 & 0.0000 & 0.0000 & 0.1994 & 0.0000 \\
\hline & 5 & 0.0000 & 0.0000 & 0.0000 & 0.0153 & 0.0000 \\
\hline \multirow[t]{6}{*}{ Irritability } & 0 & 0.8328 & 0.0000 & 0.0000 & 0.0000 & 0.0000 \\
\hline & 1 & 0.1672 & 0.0604 & 0.6206 & 0.0000 & 0.5289 \\
\hline & 2 & 0.0000 & 0.3893 & 0.3794 & 0.1462 & 0.3268 \\
\hline & 3 & 0.0000 & 0.4110 & 0.0000 & 0.5517 & 0.1443 \\
\hline & 4 & 0.0000 & 0.1394 & 0.0000 & 0.2694 & 0.0000 \\
\hline & 5 & 0.0000 & 0.0000 & 0.0000 & 0.0328 & 0.0000 \\
\hline \multirow[t]{6}{*}{ Aggression } & 0 & 1.0000 & 0.0000 & 0.5732 & 0.2581 & 0.4371 \\
\hline & 1 & 0.0000 & 0.2906 & 0.4268 & 0.0827 & 0.5168 \\
\hline & 2 & 0.0000 & 0.2289 & 0.0000 & 0.2956 & 0.0462 \\
\hline & 3 & 0.0000 & 0.4805 & 0.0000 & 0.1129 & 0.0000 \\
\hline & 4 & 0.0000 & 0.0000 & 0.0000 & 0.2506 & 0.0000 \\
\hline & 5 & 0.0000 & 0.0000 & 0.0000 & 0.0000 & 0.0000 \\
\hline \multirow[t]{6}{*}{ Dress } & 0 & 0.7583 & 0.5797 & 0.5283 & 0.9148 & 0.7521 \\
\hline & 1 & 0.2111 & 0.1375 & 0.2944 & 0.0852 & 0.1919 \\
\hline & 2 & 0.0000 & 0.1356 & 0.1773 & 0.0000 & 0.0000 \\
\hline & 3 & 0.0306 & 0.1075 & 0.0000 & 0.0000 & 0.0000 \\
\hline & 4 & 0.153 & 0.0258 & 0.0000 & 0.0000 & 0.0560 \\
\hline & 5 & 0.0031 & 0.0138 & 0.0000 & 0.0000 & 0.0000 \\
\hline
\end{tabular}


Table 2. Mean SMS-Factor 1 (Dysphoria) Scores and Sum of Items Comprising SMS Factors 2-5 (Classical Mania) for Each of the 5 GOM Groups

\begin{tabular}{lcccc}
\hline & Number Subjects & Percentage Cohort & $\begin{array}{c}\text { SMS Factor 1 } \\
\text { (dysphoria) }\end{array}$ & $\begin{array}{c}\text { SMS Factor 2-5 total } \\
\text { (classic mania) }\end{array}$ \\
\hline GOM group 1 & 67 & 20.5 & 1.194 SE 0.337 & 25.261 SE 0.849 \\
GOM group 2 & 80 & 24.5 & 1.797 SE 0.309 & 40.316 SE 0.777 \\
GOM group 3 & 59 & 18.0 & 2.161 SE 0.359 & 27.110 SE 0.905 \\
GOM group 4 & 70 & 21.4 & 8.632 SE 0.330 & 26.846 SE 0.831 \\
GOM group 5 & 51 & 15.6 & 5.833 SE 0.387 & 33.059 SE 0.974 \\
\hline
\end{tabular}

overall less psychotic, paranoid, irritable, and aggressive than Pure Type 4 episodes. Pure Type 5 episodes are second only to Pure Type 2 episodes in total severity of the classical SMS manic Factors 2-5. Overall, Pure Type 5 episodes resemble Pure Type 2 episodes but with the addition of moderate levels of dysphoria.

The analysis of GOM groups by SMS Factors (Table 3) generally confirmed the analysis by symptoms found in Table 1, and the overall patterns are more apparent. The rank order on SMS Factor 1, dysphoria, was group $4>5>2=3=1$. Groups 1, 2, and 3 had significantly lower Factor 1 dysphoria scores than groups 4 and 5, with group 4 being significantly more dysphoric even than group 5. The rank order of scores on SMS Factor 2, psychomotor acceleration, was group $2=5>1=4=$ 3 , with group 1 also $>$ group 3 . The rank order of scores on SMS Factor 3, psychosis, was group $2>3=5>4>$ 1 , with group 1 being markedly less psychotic than all other groups. The rank order of scores on SMS Factor 4, hedonic activation, was group $2=1=5=3>4$, with group 4 being clearly lower than all other groups, and 2 being greater than 3 and 5 . The rank order of scores on SMS Factor 5, irritable aggression, was group $2=4>5=$ $3>1$, with group 1 being clearly lower than all others. The rank order of severity of classical manic symptoms (sum of SMS symptoms, excluding the dysphoric symptoms) was group $2>5>3=4=1$. Table 4 displays the mean $g_{i k}$ for each of the five Pure Types by GOM group. By analysis design, the mean $g_{i k}$ of each assigned GOM group for the corresponding Pure Type is high; the percentages of total $g_{\text {ik }}$ range from $58.0 \%$ for Group 5 to $64.0 \%$ for Group 1. In contrast, the contributions from the discordant Pure Types range from $4.3 \%$ for the contribution of Pure Type 4 to Group 1, to $16.7 \%$ for the contribution of Pure Type 3 to Group 1. Data in Table 4 also emphasize that within each group, the discordant GOM Pure Types show little selective pattern of loadings. In particular, we note that Pure Types 4 and 5 do not have a particularly greater contribution to the alternate groups than do Pure Types 1-3. In other words, all five are clearly distinct groups with minor overlap.

The alignment of GOM groups with the DSM-III-R classification of manic or mixed bipolar episode is shown in Table 5. There was a significant association of DSM-III-R mixed episode with GOM group $4\left(\chi^{2}=\right.$ $126.2, \mathrm{df}=4, p<.001)$. When each combination of two GOM groups was compared by DSM-III-R subtype and a Bonferroni correction applied, group 4 had statistically more subjects meeting criteria for Bipolar Disorder, mixed, than any other group. Forty-two of the 43 episodes (98\%) meeting DSM-III-R criteria for mixed episode fell in GOM group 4 (37 cases) or group 5 (5 cases). At the same time, almost half the cases (47\%) in GOM group 4 and most cases in GOM group $5(90 \%)$ were classified as manic rather than mixed episodes by DSM-III-R. Within GOM group 4, the mean $g_{i k}$ for subjects diagnosed with Bipolar Disorder, mixed (0.642, SD 0.164 ) was not significantly different from the mean $g_{i k}$ of those diagnosed Bipolar Disorder, manic (0.564 SD $0.212, \mathrm{t}=1.739, \mathrm{df}=68, \mathrm{~ns})$.

When sex was compared across the five GOM groups, no statistical differences were noted (Table 5), however the GOM group distribution of white versus black subjects was significantly different $\left(\chi^{2}=10.874\right.$, $\mathrm{df}=4, p<.05$ ). When each combination of two GOM groups was compared by race and a Bonferroni correction applied, Pure Types 3 and 4 were statistically different $\left(\chi^{2}=8.44 \mathrm{df}=2, p<.05\right)$. Black patients were under-represented in GOM group 4 at $29 \%$ and overrepresented in GOM group 3 at 56\%. No other significant differences emerged.

\section{DISCUSSION}

Descriptive clinical subtyping of manic episodes dates to the19th century but adequately scaled, formal multivariate statistical studies are lacking. This Grade of Membership analysis resolved five Pure Types of manic episode in our sample of 327 manic inpatients. The symptom profiles of the five Pure Types have strong clinical face validity and they are also consistent with some of the subtypes described by Kraepelin.

The group loading on Pure Type 1 was quite prevalent $(20.5 \%$ of the sample). This Pure Type is a moder- 


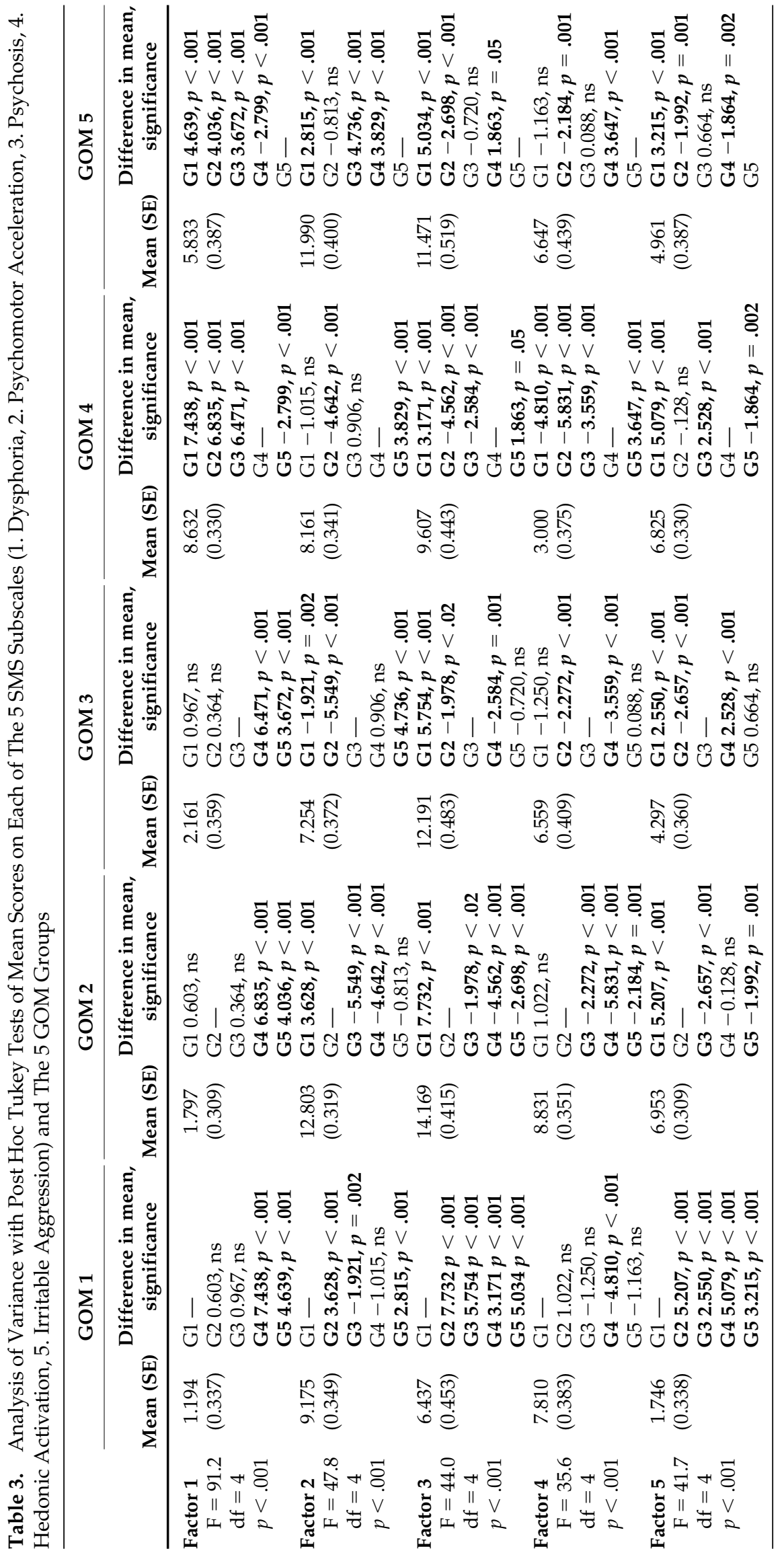




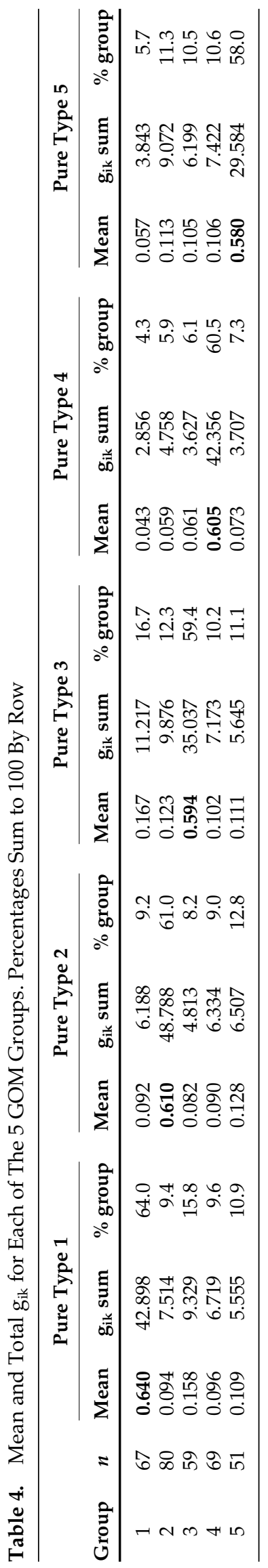

ately severe form of classical, pure mania, without psychosis and without dysphoria. It is, overall, the least symptomatic Pure Type (Table 3). It resembles the condition that Kraepelin labeled "hypomania", which he described as a mild form of mania with euphoric mood, humor, grandiosity, decreased sleep, psychomotor acceleration, and hypersexuality. Patients grouped in Pure Type 1 had a complete absence of aggression and paranoia, along with very low ratings of irritability. They were hospitalized mostly because of poor judgement and social nuisance and to ensure treatment before the episode escalated to a more severe form.

Pure Type 2, by contrast, is a very severe form of classic, pure mania, with the highest ratings on all SMS Factors except Factor 1, dysphoria. Pure Type 2 is overall the most severe subtype of mania (Table 3 ) and the most common, with $24.5 \%$ of our sample loading on this Pure Type. It is similar to "acute mania" in Kraepelin's schema, which he describes as being more severe than "hypomania," with prominent euphoria, irritability, lability, sexual drive, and grandiosity, but also high levels of psychosis, paranoia, and aggression. Patients in Pure Type 2 had the most severe denial of illness and lack of insight (Table 1). They were hospitalized, because they were clearly a danger to themselves or others through poor judgement, social nuisance, psychosis, reckless behavior, or irritable aggression, and because they were noncompliant with treatment because of their lack of insight.

Patients assigned to Group 3 also were quite prevalent at $18.0 \%$ of the sample and were intermediate in terms of overall severity (Table 3 ). This Pure Type was characterized by high ratings of psychosis, paranoia, delusional grandiosity, and delusional lack of insight, but also by lower ratings of psychomotor and hedonic activation than either Pure Types 1 or 2 . Pure Type 3 was marked by impaired insight, much like Pure Type 2, but much lower ratings of aggression and irritability. Like those in Pure Types 1 and 2, they had low ratings of dysphoria. This Pure Type resembles Kraepelin's "delusional mania," which he described as a psychotic form of mania with both grandiose and paranoid delusions that are sustained and elaborated ("which calls to mind paranoid attacks") (Kraepelin, p. 68) rather than fleeting and changeable, such as those in "acute mania." He noted that delusional mania is associated with a cheerful but labile mood, and he emphasized that excitement is not usually very severe (Kraepelin 1921, p. 70).

Patients assigned to group 4 likewise were quite prevalent at $21.4 \%$ of the sample. This Pure Type was distinguished primarily by the highest ratings on SMS Factor 1, dysphoria, and correspondingly the lowest ratings on Factor 4, hedonic activation. The total rated score on the nonmixed signs and symptoms was low, as in Pure Type 1 (Table 3). Pure Type 4 was marked by prominent depressed mood, anxiety, suicidal ideation, 
Table 5. Comparison of DSM-III-R Diagnoses of Bipolar Disorder, Manic or Mixed, Sex and Race by GOM Groups

\begin{tabular}{|c|c|c|c|c|c|c|}
\hline & GOM 1 & GOM 2 & GOM 3 & GOM 4 & GOM 5 & \\
\hline Bipolar, manic & 66 & 80 & 59 & 33 & 46 & $\chi^{2}=126.156$ \\
\hline Bipolar, mixed & 1 & 0 & 0 & 37 & 5 & $p<.001$ \\
\hline Male & 36 & 45 & 26 & 29 & 20 & $\chi^{2}=6.208$ \\
\hline Female & 31 & 35 & 33 & 41 & 31 & ns \\
\hline White & 42 & 42 & 26 & 49 & 29 & $x^{2}=10.874$ \\
\hline Black & 25 & 37 & 33 & 20 & 22 & $p<.05$ \\
\hline
\end{tabular}

and feelings of guilt. These dysphoric features were accompanied by high levels of irritability, aggression, psychosis, and paranoid thinking. It should be noted that patients grouped in Pure Type 4 also had the highest rated levels of mood lability (Table 1). They were notably more suicidal than those in other pure types. Racing thoughts and pressured speech were of moderate intensity. Humor and euphoric mood were completely absent, and ratings of grandiosity were very low. Pure Type 4 corresponds most closely with Kraepelin's "depressive or anxious mania." Kraepelin characterized this subtype by such features as a despairingly anxious mood, excitement (i.e., agitation), and ideas of sin and persecution, and he distinguished this state from "excited depression" by the presence of flight of ideas. Patients in Pure Type 4 also resemble the dysphoric, paranoid-destructive subtype described by Beigel and Murphy (1971) (Murphy and Beigel 1974). They were hospitalized not only for their manic features similar to those of patients in the first three Pure Types, but also for their depressive features and suicidal risk.

Patients assigned to Group 5 comprised $15.6 \%$ of the sample. This Pure Type also had notable dysphoric features. Depressed mood, anxiety, and lability were elevated, but the associated depressive symptoms of guilt and suicidal tendency were not. Overall, patients assigned to group 5 had significantly elevated ratings on SMS Factor 1, dysphoria, as compared with patients assigned to groups 1,2, and 3, but also significantly lower than subjects assigned to group 4 . Indeed, patients assigned to group 5 were significantly different from the other dysphoric group 4 on all five SMS Factors (Table 3). Thus, Pure Type 5 was not simply less dysphoric than Pure Type 4. In most other respects, Pure Type 5 resembled Pure Type 2 but with somewhat lower euphoria, or grandiosity, or humor, or psychosis. Nevertheless, patients in group 5 were second only to patients in group 2 in total and nonmixed mania scores on the SMS (Table 2). This presentation, with its puzzling admixture of depressed and classical manic symptoms, was recognized by both Weygandt (1901) and Kraepelin (1921). Although Kraepelin did not formalize this presentation as a group, he acknowledged that "the doctrine of mixed states is ... too incomplete for a more thorough characterization of the individual forms (Kraepelin 1921, p. 109)" and he went on to note that "cheerful and. . . . mournful or anxious mood are not simple opposites which are mutually exclusive, but .... may mix with each other in the most different ways." (Kraepelin 1921, p. 111). He further described partial mixtures, giving as an example a patient who stated that all his insides were destroyed and lacerated...(but) that the doctor might be proud to be allowed to treat him, as a case of that kind had not occurred in six hundred years (Kraepelin 1921, pp. 112-113). This blend of depressive nihilistic delusions and manic grandiose delusions is characteristic of Pure Type 5.

We call attention to the ratings of mood lability in Pure Types 4 and 5 (Table 1). Both these dysphoric Pure Types had considerable mood lability, somewhat more marked in Pure Type 4 than 5. Thus, the differences between these two dysphoric Pure Types are not attributable to a more sustained depressed mood in Pure Type 4 than 5 . The essential difference is that patients in Pure Type 4 displayed frequent alternations between a depressed mood and a nondepressed, frequently irritable mood; whereas, patients in Pure Type 5 displayed frequent alternations between a depressed mood and an elated, expansive mood. Both the dysphoric Pure Types showed prominent lability of mood, with the main difference being in the range of shortterm mood swings. In Pure Type 4, the range was from a severely depressed mood to a nondepressed or irritable mood, with minimal elation or expansiveness. In Pure Type 5, by contrast, the range was from a moderately depressed mood to a markedly elated and expansive mood.

GOM groups 4 and 5 comprised 121 patients, or 37\% of all manic episodes in our sample. In contrast, only 43 subjects ( $13 \%$ of the sample) met DSM-III-R criteria for mixed bipolar episode, and $86 \%$ of those fell in GOM group 4 . At the same time, $47 \%$ of cases in GOM group four did not qualify for the DSM-III-R diagnosis of mixed bipolar episode. There was even less correspondence between the DSM-III-R diagnoses of mixed bipolar episode and membership in GOM group 5. Only $11.6 \%$ of the DSM-III-R mixed cases fell in GOM group 5. Likewise, $90 \%$ of the 51 patients in GOM group five were not classified as in a mixed bipolar episode by 
DSM-III-R criteria. We conclude that patients in GOM group five did not qualify for the DSM-III-R diagnosis of mixed bipolar episode, because they had few of the required associated symptoms, such as guilt or suicidal tendency. Furthermore, their swings into an elated mood with hedonic activation would hinder the recognition of their periods of depressed mood. Thus, subjects in GOM group four more easily met DSM-III-R criteria for the full depressive as well as the full manic episode. Nevertheless, $47 \%$ of them did not qualify for the DSM-III-R diagnosis of mixed bipolar disorder. These data confirm the impression that DSM criteria for the mixed bipolar episode are restrictive, as we have previously concluded from factor analysis (Cassidy et al. 1998a) and receiver operating characteristic analysis (Cassidy et al. 2000).

Our results suggest that the DSM-III-R criteria for mixed bipolar disorder do not reliably identify either of the two natural groups of dysphoric manic patients revealed by this GOM analysis. Patients in both GOM groups four and five were observed to be in persistently labile episodes that did not transform into bipolar depressive episodes or into nondysphoric manic episodes. Both types clearly qualified as clinically salient mixed bipolar episodes and both were clearly different from Pure Types 1-3.

African Americans were under-represented in GOM group four and over-represented in GOM group three in this systematically evaluated cohort, suggesting that differences in manic symptom presentation exist. These differences may contribute to the clinical under recognition of bipolar disorder in African Americans.

The statistical subtypes of mania found in this GOM analysis corresponded well with many, but not all, the clinical types proposed by Kraepelin (1921). Because we did not rate cognitive features systematically, we were not in a position to recognize a group consistent with Kraepelin's "delirious mania," which has been viewed by some as rare (Goodwin and Jamison 1990, p. 24). We also did not find evidence in the GOM Pure Types of the various other mixed states described by Kraepelin and summarized by Goodwin and Jamison (1990, pp. 44-49). Those other Kraepelinian mixed subtypes likewise are uncommon and also are generally short-lived, transitional states.

In summary, this GOM analysis validated several time-honored clinical notions about the typology of manic episodes. Two major dimensions of division were found, the first between nonparanoid, nonaggressive, mostly nonpsychotic episodes (represented by Pure Type 1) and all others, and the second between dysphoric episodes (represented by Pure Types 4 and 5) and all others. The major Kraepelinian manic forms of "hypomania," "acute mania," "delusional mania," and "depressive or anxious mania" were validated. However, minor or transitional Kraepelinian subtypes, such as manic stupor, were not identified in the GOM analysis.
The Beigel-Murphy typology of EG and dysphoric PD manic subtypes was partially validated. Pure Type 1 best matches the Beigel-Murphy EG subtype; whereas, Pure Type 4 best matches their dysphoric PD subtype. However, paranoid, irritable, and aggressive features were by no means confined to Pure Type 4 . Likewise, euphoric and grandiose features often coexisted with paranoid, irritable, and aggressive features, as in Pure Types 2 and 5. These inconsistencies indicate that the Beigel-Murphy typology is incomplete, doubtless because their samples were so limited (a mere 12 patients in their first report and only 30 in their later report, (Beigel and Murphy 1971; Murphy and Beigel 1974). The GOM analysis also validates our previous finding that the SMS depression Factor 1 is orthogonal to the factors for paranoia and irritable aggression, contrary to the prediction of the Beigel-Murphy typology.

The major new finding of this GOM analysis is the recognition of a second dysphoric presentation of mania. Pure Type 4 matches the usual clinical stereotype, with severely depressed and irritable mood, associated depressive symptoms, such as guilt feelings, suicidal tendency, and psychomotor retardation, as well as paranoia and psychosis. Pure Type 5, in contrast, was less depressed, had fewer associated symptoms, and also was more classically manic than Pure Type 4 . The differences between Pure Types 4 and 5, discussed above, were statistically significant on every factor of the SMS. The DSM-III-R criteria for mixed episode aligned only with Pure Type 4, in which we found a virtual absence of euphoric mood or other signs of hedonic activation, such as humor, grandiosity, or hypersexuality. Thus, in Pure Type 4, the dominant mood was depressed with labile periods of pressured, suspicious irritability and aggression. In contrast, Pure Type 5 had considerable hedonic activation along with moderate depression, which is a more counterintuitive blend of symptoms than in Pure Type 4. In Pure Type 5, both depressed and euphoric moods were equally dominant but alternating, and the range of mood fluctuations extended further into the manic domain than was the case in Pure Type 4.

Additional studies will be needed of Pure Types 4 and 5 with alternative diagnostic criteria (Cassidy et al. 2000) and with other external validators, such as course and treatment response. Both groups will be important to study, because that will be the only way to test whether the DSM-III-R/IV requirement for the full depressive syndrome is justified. The important default hypothesis in such a test of the DSM criteria will be that a significant number of patients with mixed bipolar episodes are not recognized by current criteria, with the result that they may not receive optimal treatment. The clinical heterogeneity that this GOM analysis revealed also is a potentially significant confound in trials of an- 
timanic agents, where wide variability of response is the rule.

\section{REFERENCES}

Beigel A, Murphy DL (1971): Assessing clinical characteristics of the manic state. Am J Psychiat 128:44-50

Cassidy F, Murry E, Forest K, Carroll BJ (1997): The performance of DSM-III-R major depression criteria in the diagnosis of bipolar disorder, mixed. J Affect Disord 46:79-81

Cassidy F, Forest K, Murry E, Carroll BJ (1998a): A factor analysis of signs and symptoms of mania. Arch Gen Psychiat 55:27-32

Cassidy F, Murry E, Forest K, Carroll BJ (1998b): Signs and symptoms of mania in pure and mixed episodes. $\mathrm{J}$ Affect Disord 50:187-201

Cassidy F, Ritchie JC, Verghese K, Carroll BJ (1998c): Dexamethasone plasma concentration and cortisol response during manic episodes. Biol Pychiat 43:747-754

Cassidy F, Ahearn E, Murry E, Forest K, Carroll BJ (2000): Diagnostic depressive symptoms of the mixed bipolar states. Psycholog Med 30:403-411

Dilsaver SC, Chen YR, Shoaib AM, Swann AC (1999): Phenomenology of mania: Evidence for distinct depressed, dysphoric, and euphoric presentations. A J Psychiat 156:426-430

Double DB (1990): The factor structure of manic rating scales. J Affect Disord 18:113-119
Goodwin FK, Jamison KR (1990): Manic Depressive Illness. New York, Oxford University Press

Kendell RE (1976): The classification of depression: A review of contemporary confusion. Brit J Psychiat 129:15-28

Kraepelin E (1921): Manic Depressive Insanity and Paranoia. (Robertson GM, ed., Barclay RM, trans.) Edinburgh, Livingstone

McElroy SL, Keck PE Jr, Pope HG Jr, Hudson JI, Faedda GL, Swann AC (1992): Clinical and research implications of the diagnosis of dysphoric or mixed mania or hypomania. Am J Psychiat 149:1633-1644

Murphy DL, Beigel A (1974): Depression, elation, and lithium carbonate responses in manic patient subgroups. Arch Gen Psychiat 31:643-648

Nurnberg HG, Woodbury MA, Bogenschutz MP (1999): A mathematical typology analysis of DSM-IIIR personality disorder classification: Grade of membership technique. Comprehen Psychiat 40:61-71

Robertson GM (1890): Does mania include two distinct varieties of insanity and should it be subdivided? J Ment Sci 36:338-347

Swartz M, Blazer D, Woodbury M, George L, Lauderman R (1986): Somatization disorder in a US Southern community: Use of a new procedure for analysis of medical classification. Psycholog Med 16:595-609

Sims A (1988): Symptoms in the Mind: An Introduction to Descriptive Psychopathology. London, Bailliere Tindall

Weygandt W (1901): Ueber das manisch-depressive Irresein. Berlin Klin Wochenschr 4:105-106

Winokur G, Clayton PJ, Reich T (1969): Manic depressive illness. St Louis, C.V. Mosby 